\title{
CRITERIOS PARA LA EVALUACIÓN DE PLANES ESTRATÉGICOS DE UNIDADES ACADÉMICAS
}

\section{Introducción}

Una universidad compleja ${ }^{4}$, con un modelo de gestión semidescentralizado, debe asegurar los espacios para que cada una de las disciplinas que alberga se desarrolle conforme a su particularidad y estadios. De esta forma, se sugiere que cada unidad académica que la compone cuente con un plan de desarrollo propio, que a su vez explicite su contribución al proyecto institucional, cuyo éxito depende de un esfuerzo planificado y conjunto.

Este esquema de planificación demanda un proceso estructurado, el cual, en una de sus partes, debe evaluar la calidad, pertinencia y factibilidad del plan de desarrollo de cada unidad.

El presente trabajo tiene por objetivos:

- Proponer un conjunto de elementos a ser evaluados en el plan estratégico de una unidad académica.

- Proponer criterios de calidad para evaluar dichos elementos.

Se realizó una amplia revisión bibliográfica centrada en (1) mecanismos de evaluación de planes estratégicos y (2) en torno a la elaboración y estructura de planes estratégicos en instituciones de educación superior (IES) y su impacto.

Se llegó a una selección de siete publicaciones consideradas significativas para los efectos de este trabajo. A saber:

1. Strategic Planning for Higher Education (Kotler \& Murphy, 1981).

2. A Measurement Model of Strategic (Boyd \& Reuning-Elliott, 1998).

4 Que alberga variadas disciplinas, que imparte formación de pregrado, magíster y doctorado, y que genera conocimiento original. 
3. Planning and Performance in Public Organizations (Boyne \& GouldWilliams, 2003).

4. Strategy Development in UK Higher Education: Towards Resourcebased Competitive Advantages (Lynch \& Baines, 2004).

5. Strategic Planning, Autonomous Actions and Corporate Performance (Andersen, 2000).

6. Strategic Planning in Higher Education: who are the customers? (Conway, Mackay, \& Yorke, 1994).

7. Strategic Planning in Higher Education: a Guide for Heads of Institutions, Senior Managers and Members of Governing Bodies (HEFCE Audit Service, 2000)

Conway, Mackay \& Yorke (1994) citan en variadas ocasiones el documento Identifying the Levels of Strategy for a College or University. Long Range Planning (Shirley, 1983), razón por la cual se ha considerado también como una fuente relevante para el desarrollo de este trabajo.

\section{Propuesta de elementos y criterios para la evaluación de planes estratégicos}

\subsection{Catastro de conceptos de planificación estratégica en publicaciones revisadas}

Se realizó un catastro exhaustivo de conceptos de planificación estratégica contenidos en las publicaciones seleccionadas.

En la tabla n. ${ }^{\circ} 1$ se presentan los resultados indicando las publicaciones que mencionan explícitamente cada concepto. Aquellos recuadros que contienen dos marcas indican que esos conceptos son especialmente destacados por los autores como características positivamente correlacionadas con el desempeño futuro de la organización. 
Tabla 1: Resumen de conceptos de planificación estratégica en publicaciones

\begin{tabular}{|c|c|c|c|c|c|c|c|c|c|c|}
\hline & Conceptos & $\begin{array}{c}\text { Kotler \& } \\
\text { Murphy } \\
(1981)\end{array}$ & $\begin{array}{c}\text { Boyd \& } \\
\text { Reuning- } \\
\text { Elliott } \\
(1998) \\
\end{array}$ & $\begin{array}{c}\text { Boyne \& } \\
\text { Gould- } \\
\text { Williams } \\
(2003)\end{array}$ & $\begin{array}{c}\text { Andersen } \\
(2000)\end{array}$ & \begin{tabular}{|l|} 
Conway, \\
Mackay \\
\& Yorke \\
$(1994)$ \\
\end{tabular} & $\begin{array}{l}\text { Shirley } \\
(1983)\end{array}$ & \begin{tabular}{|c|} 
HEFCE \\
Audit \\
Service \\
$(2000)$ \\
\end{tabular} & Clasificación & Ámbito \\
\hline 1 & Misión & $\sqrt{ }$ & $\sqrt{V}$ & & $\sqrt{ }$ & $\sqrt{ }$ & $\sqrt{ }$ & $\sqrt{ }$ & & \multirow{11}{*}{$\begin{array}{l}\text { Estructura, } \\
\text { contenido y } \\
\text { proceso de } \\
\text { formulación }\end{array}$} \\
\hline 2 & Stakeholders & & & & & $\sqrt{ }$ & $\sqrt{ }$ & & MIsIon & \\
\hline 3 & $\begin{array}{l}\text { Análisis del } \\
\text { entorno }\end{array}$ & $\sqrt{ }$ & $\sqrt{ }$ & $\sqrt{ }$ & & & & $\sqrt{ }$ & & \\
\hline 4 & $\begin{array}{l}\text { Análisis de los } \\
\text { competidores }\end{array}$ & & $\sqrt{ }$ & & & & $\sqrt{ }$ & $\sqrt{ }$ & Análisis entorno & \\
\hline 5 & \begin{tabular}{|l|} 
Análisis de los \\
recursos
\end{tabular} & $\sqrt{ }$ & & $\sqrt{ }$ & & & & $\sqrt{ }$ & Análisis interno & \\
\hline 6 & Visión & $\sqrt{ }$ & $\sqrt{ } \sqrt{ }$ & $\sqrt{ }$ & $\sqrt{ }$ & & $\sqrt{ }$ & $\sqrt{ }$ & Visión & \\
\hline 7 & \begin{tabular}{|l|}
$\begin{array}{l}\text { Formulación de } \\
\text { la estrategia }\end{array}$ \\
\end{tabular} & $\sqrt{ }$ & & $\sqrt{ }$ & & & & $\checkmark$ & \multirow{3}{*}{$\begin{array}{l}\text { Estrategias y } \\
\text { acciones }\end{array}$} & \\
\hline 8 & $\begin{array}{l}\text { Programas } \\
\text { y servicios } \\
\text { ofrecidos } \\
\end{array}$ & & & & & & $\sqrt{ }$ & & & \\
\hline 9 & \begin{tabular}{|l|} 
Planes de \\
Acción de corto \\
plazo
\end{tabular} & & $\sqrt{ } \sqrt{ }$ & $\sqrt{ }$ & & & & $\sqrt{ }$ & & \\
\hline 10 & \begin{tabular}{|l|} 
Metas de \\
objetivos largo \\
plazo
\end{tabular} & $\sqrt{ }$ & & $\sqrt{ }$ & $\sqrt{ }$ & & $\sqrt{ }$ & $\downarrow$ & \multirow{2}{*}{ Metas } & \\
\hline 11 & \begin{tabular}{|l|}
$\begin{array}{l}\text { Objetivos } \\
\text { anuales }\end{array}$ \\
\end{tabular} & & $\sqrt{ }$ & $\sqrt{ }$ & $\sqrt{ }$ & & $\sqrt{ }$ & & & \\
\hline 12 & \begin{tabular}{|l|} 
Plan de \\
desarrollo de \\
recursos y áreas \\
estratégicas \\
\end{tabular} & 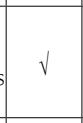 & & $\sqrt{ }$ & & & $\sqrt{ }$ & $\sqrt{ }$ & \multirow{4}{*}{ Implementación } & \multirow{9}{*}{$\begin{array}{l}\text { Viabilidad, } \\
\text { implementación } \\
\text { y seguimiento }\end{array}$} \\
\hline 13 & \begin{tabular}{|l|} 
Desarrollo \\
de recursos \\
humanos \\
\end{tabular} & & & & & & $\sqrt{ }$ & $\sqrt{ }$ & & \\
\hline 14 & \begin{tabular}{|l|}
$\begin{array}{l}\text { Diseño de la } \\
\text { organización y } \\
\text { sistemas }\end{array}$ \\
\end{tabular} & $\sqrt{ }$ & & & & & $\sqrt{ }$ & $\sqrt{ }$ & & \\
\hline 15 & \begin{tabular}{|l|} 
Delegación y \\
flexibilidad \\
para el ajuste \\
del Plan \\
\end{tabular} & & & & $\sqrt{ }$ & & & & & \\
\hline 16 & \begin{tabular}{|l|} 
Financiamiento \\
(obtención \\
de ingresos o \\
reducción de \\
costos) \\
\end{tabular} & & & & & & & $\sqrt{ }$ & \multirow{3}{*}{ Viabilidad } & \\
\hline 17 & $\begin{array}{l}\text { Actitud de } \\
\text { miembros hacia } \\
\text { el plan }\end{array}$ & & & $\sqrt{ }$ & & & & & & \\
\hline 18 & $\begin{array}{l}\text { Capacidad, } \\
\text { de liderazgo, } \\
\text { política y } \\
\text { gestión del } \\
\text { poder } \\
\end{array}$ & & & $\sqrt{ }$ & & & & $\sqrt{ }$ & & \\
\hline 19 & $\begin{array}{l}\text { Gestión de la } \\
\text { información }\end{array}$ & & & $\sqrt{ }$ & & & & $\sqrt{ }$ & \multirow[b]{2}{*}{ Seguimiento } & \\
\hline 20 & \begin{tabular}{|l|} 
Seguimiento y \\
evaluación de \\
directivos
\end{tabular} & & $\sqrt{ } \sqrt{ }$ & & & & & $\sqrt{ }$ & & \\
\hline
\end{tabular}


Para sistematizar y organizar el análisis, estos conceptos se han clasificado en nueve títulos (misión, análisis del entorno, análisis interno, visión, estrategias y acciones, metas, implementación, viabilidad y seguimiento), los que a su vez se han organizado en dos ámbitos: (1) estructura, contenido y proceso de formulación, y (2) viabilidad, implementación y seguimiento.

Si bien los conceptos presentados en la tabla n. ${ }^{\circ} 1$ son bastante exhaustivos en cuanto al contenido e implementación de un plan estratégico, ellos no entregan guías para la reflexión en torno a los fundamentos sobre los cuales la estrategia se sustenta; ¿cuáles son las ventajas competitivas?, ¿cómo se hacen sustentables en el tiempo?

Lynch \& Baines (2004), analizando la situación de la IES en el Reino Unido (RU), que se han visto enfrentadas a escenarios de mayor competencia, proponen la aplicación del sistema RBV (resourced-based view) para este tipo de instituciones. Este concepto busca identificar conjuntos de recursos particulares de la organización que le permiten lograr un desempeño superior y establecer una ventaja competitiva sustentable, es decir, se hace difícil imitarla y no es negociable por otra institución.

A su vez, Conway, Mackay \& Yorke (1994), aludiendo a la misma situación de competencia a la que se han visto enfrentadas las IES en el Reino Unido (RU) posterior a la reforma del año 1988, destacan la importancia de identificar los clientes y los caminos para satisfacerlos adecuadamente. De esta forma, se propone un tercer ámbito de reflexión sobre los planes estratégicos, cual es, (3) los fundamentos de la estrategia y ventajas sostenibles.

\subsection{Elementos y criterios de evaluación}

Para cada uno de los ámbitos de evaluación definidos se fundamenta una propuesta de elementos y criterios de evaluación. 
2.2.1 Estructura, contenido y proceso de formulación de un plan estratégico

Para cada uno de los elementos presentados en la columna "clasificación" de la tabla n. ${ }^{\circ} 1$ se argumentan el conjunto de criterios que nos permitirán evaluarlos.

\subsubsection{Misión}

Kotler \& Murphy (1981) definen la Misión como el propósito básico y fundacional de la institución, que establece nuestros ámbitos de acción. Ésta "actúa como una mano invisible que guía a los distintos integrantes de la institución a trabajar independientemente y, aún así, colectivamente hacia el logro de los objetivos de la organización". Una Misión efectiva es aquella que está orientada a las necesidades del medio, que es factible, motivadora y específica.

Por su parte, Boyd \& Reuning-Elliott (1998) identifican los factores claves de una estrategia: declaración de misión, análisis de tendencias, de competidores, objetivos de largo plazo, anuales, planes de acción de corto plazo y evaluación en la marcha. A partir de estos factores administraron una encuesta a altos ejecutivos de hospitales, quienes dieron el mayor énfasis a los objetivos anuales, seguidos de los de largo plazo, la declaración de misión y el plan de acción de corto plazo. Si bien los autores no dan luces respecto a lo que debe ser una Misión y su contenido, sí le dan valor como uno de los componentes importantes de un plan estratégico.

Andersen (2000) bosqueja una perspectiva integradora entre la planificación estratégica y las acciones autónomas. Constata la relación positiva entre la planificación y el desempeño de las instituciones. Sin embargo, propone un efecto valedero de las acciones autónomas sobre el de la organización, en especial por la vía de la innovación. En efecto, grandes estrategias podrían surgir sin la conciencia explícita de los directivos superiores (Bower, 1970/1982; Normann \& Pennings, 1985; Burgelman, 1983; Burgelman, 1988; Mintzberg, 1994; Noda $\&$ Bower, 1996).

Además, Andersen (2000) declara que la habilidad de los gerentes para tomar decisiones en forma autónoma e independiente 
permite a la organización contar con una mayor capacidad de respuesta a los cambios en el medio, especialmente beneficioso en un contexto complejo y cambiante.

En conclusión, debemos considerar que los planes estratégicos de unidades académicas, si bien deben declarar una adscripción explícita y concreta a los desafíos institucionales de la universidad, deberían contener aspiraciones propias, identificadas con acciones específicas para el desarrollo de la disciplina cultivada por la unidad. Ello favorecerá la capacidad de adaptación, la diversidad de soluciones a desafíos y problemáticas particulares y espacios para la generación de decisiones autónomas. Esto sugiere contar con una Misión y Visión propias, que expliciten los propósitos básicos de la unidad y objetivos, refuercen su identidad y permitan a las autoridades tomar decisiones propias y autónomas dentro de este contexto de referencia.

Conway, Mackay \& Yorke (1994) le asignan mucha importancia a la Misión dentro de los planes estratégicos y la explicitación de los stakeholders en esta declaración. La describen como el propósito perdurable de la organización, que la distingue de otras y define los alcances de su accionar. Adicionalmente, refleja la imagen que la institución quiere proyectar y la que tiene de sí misma, identificando las principales áreas de servicios a las cuales se dedica y reconociendo la demandas legítimas de sus stakeholders (Pearce, 1982). Shirley (1983) -citado por Conway, Mackay \& Yorke- sugiere seis áreas críticas de decisión entre las cuales se cuenta la definición de sus stakeholders y su Misión.

Por su parte, el Higher Education Funding Council for England (HEFCE Audit Service, 2000), en conjunto con los directivos de varias universidades, elaboró una guía para la planificación estratégica en el año 2000, la cual coincide con los inicios de la reforma del sistema de educación superior del año 1988.

Destaca en este documento el sentido trascendente dado a la planificación estratégica, reflejado en declaraciones tales como "dado que las circunstancias cambian, la planificación estratégica permite a las instituciones responder en forma coherente y consistente con 
las directrices que se ha propuesto, más que reaccionar en forma adecuada a las oportunidades y riesgos que va enfrentando". Es decir, esta programación evita reacciones antojadizas producto de hechos inesperados y que pudieran no ser compatibles con la identidad de la institución. Las declaraciones en sí mismas refuerzan la importancia y necesidad de establecer una Misión.

Este documento plantea la planificación estratégica como un proceso cíclico cuyas etapas son planificación, documentación e implementación y monitoreo.

En la etapa de planificación (llamémosla de formulación del plan) se propone como primer paso la exploración, orientada a identificar los factores que podrían afectar a la organización y su posición, compuesta a su vez por: análisis del entorno, evaluación de los recursos internos e identificación de los mandatos que reafirman el propósito de la institución (Misión).

De esta forma, se propone que los criterios de evaluación de la Misión consideren que esta...

a. Tenga una razón de ser propia, basada en una disciplina específica o bien en un quehacer particular que la distingue de las demás y que, en definitiva, explicita el propósito básico y fundacional de la unidad

b. Identifique sus principales áreas de servicios y stakeholders

c. Se alinee con la Misión de su universidad

d. Sea factible, motivadora y específica.

\subsubsection{Análisis del entorno}

Kotler \& Murphy (1981) sugieren un análisis que entienda e incorpore en el proceso de reflexión el contexto en el cual la institución se desenvuelve y cuyas características pueden afectar de alguna forma su accionar. Algunos ámbitos de exploración sugeridos son: el interno (relativo a la institución pero que no depende de la unidad en análisis), el mercado (para cada uno de los ámbitos de acción de la institución), la competitividad, lo público y el macro entorno (desarrollo demográfico, económico, tecnológico, político y cultural). 
Si bien Boyd \& Reuning-Elliott (1998) consideran el análisis del entorno y de competencia, estos no son factores críticos de un plan estratégico. Ello nos sugiere moderar nuestras posiciones respecto a la existencia o no de este tipo de análisis.

Boyne \& Gould-Williams (2003), basados en investigaciones anteriores (Camillus, 1975; Capon, Farley, \& Hubert, 1987; Kay, 1993; Self, 1974; Leach, 1982), señalan algunas causas por las cuales los procesos de planificación tendrían impactos positivos en el desempeño organizacional, argumentos que nos permitirán discriminar, a su vez, sobre las características de calidad de algunos contenidos de un plan. Entre otros, la planificación permite identificar acontecimientos externos y cambios internos e incorporarlos dentro de la estrategia para tomar decisiones informadas sobre diferentes acciones más que obedeciendo a intuiciones. En efecto, esos autores aseguran que la preocupación sobre la calidad de un plan no sólo debe estar puesta en este mismo, sino también en el proceso que lo llevó a su formulación, en tanto un plan comprensible no asegura que el proceso que lo generó sea igualmente claro y coherente en su análisis, así como un análisis y formulación comprensible no necesariamente es capaz de generar un plan claro. Finalmente, otros autores destacan la gravitación que tiene sobre el desempeño la actitud de los miembros de la organización ante éste (Armstrong, 1982).

Para la realización de su estudio, Boyne \& Gould-Williams (2003) proponen como medidas para planificar: la fijación de metas u objetivos, análisis externo e interno, plan de acción y la percepción sobre el proceso mismo ${ }^{5}$. Una de las conclusiones recogidas por los autores es que todo esto tiene un impacto neutro sobre el desempeño de la institución. En definitiva, más importante que el proceso de planificación y la reflexión sobre las capacidades internas y el entorno que éste considera, es la valoración (actitud positiva y motivada) que la comunidad le confiere al proceso de formulación.

Por su parte, el HEFCE Audit Service (2000) considera que en tanto las circunstancias cambian, la planificación hace posible que

5 Principalmente dado por la facilidad o complejidad en la recolección de la información y en la utilidad de la misma. 
las instituciones den respuesta a ellas de manera consistente con sus propias directrices, más allá de la reacción puntual a las oportunidades y riesgos de la coyuntura. En la etapa de planificación, se propone la exploración de los factores que podrían afectar a la organización y su posición, entre ellos el análisis del entorno, para lo cual sugiere examinar la posición de mercado respecto de otras instituciones, a partir de información pública o bien especialmente elaborada para el efecto, debiendo este examen desarrollarse en torno a dos criterios, el académico y el económico.

Desde esta perspectiva, la propuesta de criterios para la evaluación del análisis del entorno considera que esta...

a. Incluye a la misma institución en todo aquello que no es controlable por la unidad y aborda la contingencia normativa, legal, social, económica y cultural, tanto en el ámbito nacional como internacional.

b. Contempla la oferta de otras instituciones del medio que constituyen una opción, basada en perspectivas académicas y económicas.

c. Destaca elementos que afectan el quehacer de la unidad e incide efectivamente en la definición de las estrategias.

d. Considera la opinión de externos y stakeholders, haciendo partícipe de este análisis a la comunidad.

\subsubsection{Análisis interno}

Kotler \& Murphy (1981) proponen un análisis de los recursos internos de la organización. En este sentido, la estrategia debe ser coherente con lo que la institución es, considerando el desarrollo de aquellas cosas que la limitan o bien aprovechando las que la potencian. Desde esta perspectiva, algunos ámbitos de exploración son: personal, financiamiento, facilidades y sistemas.

Al igual que en el caso del análisis externo, de la lectura de Boyne \& Gould-Williams (2003) se puede concluir que más relevante que el proceso de planificación y del análisis interno, es que la comunidad enfrente el proceso con una actitud positiva y motivada, lo cual, por supuesto, no invalida la necesidad de realizar dicho análisis. En efecto, 
la medición de la percepción sobre el proceso de planificación está basada en el mismo ejercicio que comprende el análisis interno y, por tanto, en el grado de participación de la comunidad.

De la declaración del HEFCE Audit Service (2000) en torno al proceso de planificación y la importancia del diagnóstico, destaca un adecuado análisis de las competencias y capacidades internas. De esta forma, en la etapa de planificación, éste propone la evaluación de los recursos internos a la luz de los objetivos que la institución persigue.

Por otra parte, si bien Conway, Mackay \& Yorke (1994) no hablan explícitamente sobre el análisis interno, al declarar la necesidad que tuvieron las universidades del RU de focalizarse en sus clientes para afrontar mejor un ambiente competitivo, expresan la necesidad de que el análisis interno también tome como referencia a los stakeholders y las formas para satisfacer los requerimientos de cada uno de ellos.

Así visto, la propuesta de criterios para la evaluación del análisis interno considera que este:

a. Cuenta con un análisis de los recursos disponibles a la luz de las demandas de la visión propuesta, los requerimientos de los stakeholders y la exploración de la competencia realizada.

b. Es exhaustivo en términos de abordar todos aquellos recursos que se consideran necesarios para constituir la oferta deseada (infraestructura, tecnologías, personal académico y administrativo, organización y gobierno y procesos)

c. Ha considerado la opinión de los stakeholders y ha hecho participar a la comunidad en este análisis.

\subsubsection{Visión}

Kotler \& Murphy (1981), dentro de un concepto amplio, describen lo formulación de objetivos en tres niveles: la Misión, los objetivos y los desafíos. Siendo de mayor especificidad los objetivos ${ }^{6}$ deben estar en sintonía con la Misión. Además, es importante tener presente las competencias y tensiones que pueden existir entre los distintos objetivos, tanto por las naturales limitaciones de financiamiento -que

6 Lo que se interpreta como lo que comúnmente llamamos Visión. 
imponen una priorización- como por los trade-offs existentes entre unos y otros. Los desafíos, por su parte, son interpretados como las metas, por medio de las cuales se establecen magnitudes, plazos y responsabilidades sobre los proyectos del Plan.

Por su parte, Boyd \& Reuning-Elliott (1998) incluyen los objetivos de largo plazo entre los factores a considerar en su estudio, recayendo en éstos el segundo mayor énfasis puesto por los ejecutivos entrevistados. No obstante lo anterior, los autores no agregan mayor información sobre posibles criterios para considerar en su evaluación, más que su sola presencia en el Plan.

Boyne \& Gould-Williams (2003) señalan que la planificación fuerza a los ejecutivos a clarificar sus objetivos y, por esa razón, entrega un contexto de asignación de recursos en orden a los propósitos de la organización. Adicionalmente, provee objetivos que pueden ser comunicados a todo el cuerpo directivo y así ajustar su esfuerzo de acuerdo a ellos. Sin embargo, su estudio concluye que una cantidad desmedida de éstos se asocia con un menor desempeño futuro de la organización. Una de las posibles explicaciones es que la cantidad mayor de objetivos induce a la confusión entre las personas que conforman la institución.

Andersen (2000) declara que la habilidad de los gerentes para tomar decisiones en forma autónoma e independiente permite a la organización contar con una mayor capacidad de respuesta a los cambios en el medio, especialmente beneficioso cuando se encuentra en un contexto complejo y cambiante. De esta forma, como ya se analizó para el caso de la Misión, contener aspiraciones propias, que se identifiquen con acciones que serían específicas para el desarrollo de la disciplina cultivada por la unidad y que podrían no ser las mismas para otras, entrega los espacios necesarios para que se den procesos de toma de decisiones autónomos y coherentes con el conjunto de la institución. Ello sugiere la necesidad de contar con una Visión propia, que explicite los objetivos que se propone la unidad a partir de su situación actual. En sintonía con lo anterior, Shirley (1993) señala la conveniencia de fijar objetivos y metas dentro de las áreas críticas de decisión de un plan estratégico. 
En la etapa de documentación del plan, la HEFCE Audit Service (2000) menciona varios documentos con diversos objetivos y públicos. En este contexto, sugiere como uno de los contenidos relevantes de incluir en aquellos documentos que resulten de la planificación, la declaración de una Visión de largo plazo.

Como se verá en el capítulo 2.2.3, la participación de los stakeholders en el proceso de formulación de la estrategia es fundamental, al punto que la Visión propuesta debe orientarse a la satisfacción de las aspiraciones que éstos tienen sobre lo que la institución le ofrece.

Por último, al estar una unidad académica inserta en un contexto organizacional más amplio, su Visión necesariamente debe contribuir a los objetivos de largo plazo que la institución como un todo se ha propuesto.

De esta forma, los criterios propuestos para evaluar la Visión estiman que esta:

a. Propone objetivos de largo plazo, que proyecten a la unidad en el tiempo y reflejen la oferta que desea entregar a los stakeholders.

b. Incluye no más de siete elementos y ellos no se contraponen entre sí.

c. Es coherente y contribuye con el Plan de Desarrollo Institucional.

d. Posee elementos claros y fáciles de transmitir, y contiene aspiraciones propias que reflejan el sentir de la comunidad, la que se ha hecho partícipe en su elaboración.

\subsubsection{Estrategias y acciones}

Entre las etapas que Kotler \& Murphy (1981) sugieren en el proceso de planificación está la elaboración de la estrategia. Ella se define a partir de las aspiraciones que la comunidad propone en la Visión y las ofertas de valor que pretende ofrecer a sus stakeholders. Todo lo anterior, a la luz de las carencias detectadas al interior de la institución y del análisis del entorno realizado. Por su parte, Boyd \& Reuning-Elliott (1998) señalan que los planes de acción de corto plazo constituyen otro elemento importante de evaluar en los planes estratégicos, y que estarían dentro de los más relevantes según la encuesta administrada a altos ejecutivos en su estudio. 
Dado que el plan estratégico permite tomar decisiones sobre diferentes cursos de acción a seguir, basados en información comprendida más que en meras intuiciones, Boyne \& Gould-Williams (2003) proponen al plan de acción como una de las medidas de la planificación para evaluar en su investigación. Sin embargo, el mismo trabajo concluye que la presencia de los planes de acción tendría un impacto neutro sobre el desempeño de la institución.

Shirley (1983) incluye dentro de las seis áreas críticas de decisión en un plan estratégico los programas y servicios ofrecidos, el área geográfica del accionar de la institución, las ventajas comparativas vistas sobre otras instituciones del sector y, producto de lo anterior, los recursos, estructura organizacional, formas de gobierno y desarrollo de recursos humanos.

El HEFCE Audit Service (2000) propone como una de las etapas del proceso de planificación la generación de ideas orientadas a realizar nuevas actividades, mejoras a otras o bien descontinuar selectivamente aquellas que lo justifiquen, adquiriendo gran valor en este punto la capacidad de gestionar la innovación, además de la facilitación, entendida como el análisis y disposición de los recursos y capacidades necesarias para hacer viable el plan. Adicionalmente, entre la documentación final del Plan, HEFCE indica la necesidad de incorporar un resumen de las estrategias, integradas con las actividades y recursos, debiendo las tareas específicas asignarse a responsables individuales y ser limitadas en su cantidad.

De esta forma, los criterios propuestos para evaluar estrategias y acciones consideran que éstas:

a. Sean coherentes con la Misión, la Visión y el análisis realizado (orientadas a superar las debilidades de la unidad, aprovechar las fortalezas, superar eventuales amenazas o aprovechar oportunidades que el medio ofrece).

b. Sean concretas y se proyecten en el corto plazo mediante una programación de actividades para el año siguiente.

c. Posean una oferta de servicios para reforzar, crear o eliminar, sobre la base del análisis realizado. 


\subsubsection{Metas}

Kotler \& Murphy (1981), dentro de lo que es la formulación de los objetivos, plantean como definición de desafíos lo que entendemos como metas, estableciendo a través de ellos magnitudes, plazos y responsabilidades sobre los proyectos del plan. Boyd \& ReuningElliott (1998), por su parte, proponen, dentro de los aspectos medibles en una planificación estratégica, los objetivos anuales, elemento al cual los altos ejecutivos de entrevistados en el estudio dieron el mayor énfasis. De manera similar, Boyne \& Gould-Williams (2003), plantean la fijación de metas u objetivos como elemento de evaluación de un plan estratégico. Shirley (1983) también sugiere, dentro de sus seis áreas críticas de decisión en un plan estratégico, la declaración de objetivos y metas.

Por su parte, el HEFCE Audit Service (2000) propone la conveniencia de explicitar tareas y metas (específicas, medibles, logrables, relevantes y oportunas) o bien hitos, durante la etapa de documentación, señalando también la conveniencia de formular metas de mediano plazo.

En consecuencia, los criterios propuestos para la evaluación de las metas contemplan que:

a. La Visión tenga asociado un conjunto de metas expresadas en indicadores o hitos, los que, mediante la valorización o explicitación de un suceso en un plazo determinado, darán cuenta del impacto que el Plan ha suscitado en éste (indicadores de impacto); presentan además valorizaciones anteriores al plazo del Plan tomadas como referencia.

b. Para cada una de las acciones principales de la estrategia ha establecido un responsable, indicadores e hitos que permitirán constatar su realización, con metas anuales y plazos claros.

\subsubsection{Viabilidad, implementación y seguimiento}

Así como la bibliografía destaca como elementos fundamentales, para que se transformen en herramientas con un impacto efectivo en el desarrollo de la institución a la estructura y contenido de los planes, también lo hace con la capacidad de gestionar, liderar, comunicar y realizar el seguimiento de éstos. 
Como se observará, existen varios elementos de evaluación que bien podrían pertenecer al ámbito de "estructura, contenido y proceso de formulación" o bien al de "viabilidad, implementación y seguimiento" . Sin embargo, dado que estas clasificaciones son utilizadas para la estimación de los planes, se considerarán estos elementos en uno de los dos ámbitos para no repetir su evaluación.

\subsubsection{Viabilidad}

Boyne \& Gould-Williams (2003), queriendo demostrar los impactos positivos de la planificación en el desempeño de las instituciones públicas, toman como referencia lo ya hecho en el sector privado. En efecto, se ha comprobado su buen resultado en el trabajo institucional, lo cual se debería, entre otras razones, a que la planificación exige que los ejecutivos clarifiquen sus objetivos, permitiendo que éstos sean efectivamente comunicados a todo el cuerpo directivo y de esta forma alineando su accionar, constituyéndose en una potente herramienta para ejercer liderazgo.

Por otra parte, Boyne \& Gould-Williams (2003) recogen una serie de críticas en torno a la aplicación de planificación estratégica a instituciones públicas, entre ellas, que los planes logran que otros hagan lo que de otra forma no harían, lo que implica una gran capacidad política y administración del poder (Wildavsky, 1973). Estas críticas permiten reflexionar sobre la necesidad de que conjuntamente a la formulación de un plan estratégico y su implementación, se deben estimar las capacidades políticas y de liderazgo, a través del desarrollo de documentos, medios adecuados de comunicación, institucionalidad, entre otros.

Adicionalmente, Boyne \& Gould-Williams (2003) hacen referencia a que los impactos del Plan en el desempeño de la institución también están determinados por la actitud interna de los miembros hacia la herramienta (Armstrong, 1982), lo que confirma la necesidad de contar con capacidades de liderazgo y comunicación adecuada y pertinente de éste. Tanto es así, que posteriormente el

7 También podría darse dentro del ámbito de fundamentos de la estrategia y ventajas sostenibles. 
paper postula a la "percepción sobre el proceso de planificación" como una medida para considerar en el estudio, de la cual se concluye su impacto positivo sobre el desempeño.

El documento Strategic planning in higher education: a guide for heads of institutions, senior managers and members of governing bodies (HEFCE Audit Service, 2000) entrega propuestas concretas en torno a las capacidades de gestión, implementación y seguimiento de un plan, entre las cuales ofrece importantes luces sobre los factores de viabilidad. El mismo documento define la planificación como un proceso cíclico, cuyas etapas son la planificación propiamente tal, la documentación y la implementación y monitoreo. Para la etapa de implementación se presentan características relacionadas con el ejercicio del liderazgo y la gestión del proyecto, esenciales para una estrategia innovadora, tales como: capacidad de gestión del cambio (comunicar, consultar, guiar, desarrollar y retroalimentar); contar con equipos para la gestión de los proyectos; asumir riesgos razonables, (capacidad de gestionarlos y tolerancia a fallar).

En el caso de la etapa de monitoreo, también se menciona una característica sobre la capacidad de liderazgo en el seguimiento del plan, que se refiere al rastreo regular y periódico que aplica el equipo directivo superior.

Para la etapa de planificación, el documento señala la relevancia que tiene el liderazgo en la definición e implementación del Plan. El directivo que lo ejerce debe contar con la capacidad de visualizar, consultar, emplazar y comunicar. Adicionalmente se propone una serie de facilitadores que hagan viables sus propuestas; entre ellas se menciona la gestión financiera como una de las habilidades requeridas para la planificación e implementación del cambio, como asimismo la generación de ingresos y los incentivos a la reducción de costos.

Por último, si bien la administración central de las universidades podría concentrar una cantidad importante de recursos y su asignación, no es menos cierto que las principales capacidades de generación están instaladas en las unidades académicas. De esta forma, es fundamental que ellas se hagan parte del desarrollo e 
implementación de estrategias de financiamiento, cuidando que no se creen incoherencias entre fuentes, que pudieran traducirse en el menoscabo de alguna de ellas.

En consecuencia, como criterios para evaluar la viabilidad del plan, se propone que este:

a. Plantee una estrategia de financiamiento razonable tanto en relación con los recursos que la propia unidad pretende obtener (y la forma en que lo hace), como a los solicitados a las fuentes financieras que la institución ofrece.

b. No evidencie incompatibilidades entre las formas de financiamiento propuestas para algunas acciones y otras actividades que plantea.

c. Represente una comunidad cohesionada en torno a las propuestas y una actitud proactiva en torno a ellas.

d. Cuente con directivos dotados de la capacidad política y del liderazgo necesario para llevar con éxito la implementación.

\subsubsection{Implementación}

Kotler \& Murphy (1981) postulan la necesidad de hacer un análisis de los recursos ${ }^{8}$ requeridos para llevar adelante la estrategia, establecer claramente las metas ${ }^{9}$ asociadas a los objetivos que el Plan propone y reparar en el diseño organizacional y de los sistemas o procesos que permitan su implementación. Algo similar ya lo proponía Chandler (1962), al decir que "la estructura debe seguir la estrategia".

Boyne \& Gould-Williams (2003), queriendo demostrar los impactos positivos de la planificación en el desempeño de las instituciones públicas, toma como referencia experiencias del sector privado. En efecto, señalan que se ha probado dicho impacto en el desempeño de las instituciones privadas, lo cual se debería, entre otras razones, a que obliga a los ejecutivos a clarificar sus objetivos, razón por la cual provee un contexto de asignación de recursos en

8 Personal, financiamiento, facilidades y sistemas.

9 Magnitudes, plazos y responsables. 
orden a los propósitos de la organización ${ }^{10}$. Esto no muestra tan sólo la importancia de que los planes cuenten con una declaración de objetivos, sino también, desde la perspectiva de la implementación, que el desarrollo de recursos sea coherente con los desafíos que el Plan se propone.

Andersen (2000) postula que si bien la planificación estratégica formal genera efectos positivos en el desempeño de las organizaciones, las acciones autónomas de las unidades o reparticiones de la institución permiten que la organización cuente con una mayor capacidad de respuesta en el medio. De hecho, la planificación genera un pensamiento adaptativo estratégico a través de los directivos generando una coordinación entre ellos.

Más allá de la razonable discusión que podría surgir al interior de la institución en relación con el grado de autonomía que ella entrega a sus unidades académicas en los procesos de toma de decisiones, la flexibilidad, expresada en las capacidades de gestión y seguimiento, debiera ser una característica deseable al interior de los planes estratégicos, de tal forma que las responsabilidades sobre metas y objetivos estén convenientemente distribuidas entre los directivos de la unidad; se consideren sistemas de seguimiento en el tiempo y se suscriba un compromiso con la comunidad académica de hacer una revisión y ajuste anual de sus estrategias. Finalmente, que la unidad académica exprese la intención y capacidad de monitorear indicadores e información clave sobre los avances y los elementos significativos del ambiente, que eventualmente podrían determinar ajustes a la estrategia, así como las condiciones de calidad académica que la IES no está dispuesta a comprometer en el proceso de implementación de ésta.

En este sentido, Shirley (1983) propone que después de haber resuelto una estrategia, la institución debe pensar en los recursos que serán necesarios para su implementación, la estructura organizacional, las formas de gobierno y el desarrollo de capital humano. La HEFCE

10 No es el espíritu de este trabajo ni la razón por la cual está citada esta referencia. En todo caso, tal como lo presenta posteriormente esta publicación, son algo aceptado los impactos positivos de la planificación en instituciones públicas. 
Audit Service (2000), para la etapa de implementación sugiere algunos elementos que favorecerían la ejecución del plan, tales como son contar con una estructura organizacional acorde a la estrategia y utilizar consultores como agentes de cambio.

Si bien las etapas de planificación y documentación del proceso no son explícitamente gestión, implementación y monitoreo, entregan luces importantes para ellos. Por ejemplo, en la propuesta de facilitadores para la implementación del Plan se estima necesario comprender las necesidades y desarrollo de sistemas de información como una habilidad importante para la implementación del cambio, así como contar con sistemas y tecnologías de la información, desde la perspectiva de los recursos. En la etapa de documentación se establece la presencia de ciertas estrategias focalizadas en recursos transversales o áreas de desarrollo: estrategia académica, de investigación, de enseñanza y aprendizaje; estrategia financiera, de recursos humanos y tecnologías de la información. Considerando la importancia del cuerpo académico en la oferta de valor de la universidad y su significancia en la estructura de costos, bien vale contemplar un plan de desarrollo del grupo docente, como elemento superlativo en la evaluación de la planificación de una unidad académica.

Conforme a lo antes señalado, los criterios que se proponen para evaluar los elementos de implementación contenidos en un plan, estipulan que este:

a. Cuenta con una estimación de recursos coherente con los desafíos que se plantea o con las carencias con que cuenta la unidad.

b. Considera un plan de desarrollo de la planta de profesores que explicita su evolución en el tiempo.

c. Propone la elaboración de un plan de desarrollo físico, de tecnologías, de equipamiento y en torno a áreas consideradas estratégicas para el futuro de la unidad.

d. Demuestra la autonomía y flexibilidad necesaria para ajustar las actividades y financiamiento si es que las condiciones lo ameritan.

e. Plantea la necesidad de ajustar -o bien proponer- el diseño organizacional y de los sistemas de acuerdo a los requerimientos de la estrategia. 


\subsubsection{Seguimiento}

Boyd \& Reuning-Elliott (1998), en su paper A Measurement of Strategic, concluyen que entre los elementos más gravitantes en la calidad de una programación están los objetivos anuales y el plan de acción de corto plazo (Boyd \& Reuning-Elliott, 1998), los cuales, dadas su especificidad y concreción, son un aporte a las capacidades de gestión y seguimiento del Plan. Como ya se ha señalado, las evaluaciones sobre la marcha ${ }^{11}$ poseen una alta correlación con todos los atributos considerados más significativos.

Por su parte, Boyne \& Gould-Williams (2003), al recoger una serie de críticas en torno a la aplicación de planificación estratégica a instituciones públicas, mencionan entre ellas que los procesos formales requieren contar con una gran cantidad de información, además de habilidades para poder extraer conclusiones e incorporarlas dentro de la correspondiente planificación, lo cual lo hace tremendamente dificultoso (Streib \& Poister, 1990). Esta y otras críticas permiten reflexionar sobre la necesidad de que conjuntamente a la formulación de un plan estratégico y su implementación se debe prever la capacidad de generar y administrar en forma eficiente y efectiva la información necesaria para la reflexión y seguimiento del desempeño.

Con respecto a la primera etapa (planificación) el HEFCE Audit Service (2000) propone una serie de facilitadores que harían viables las propuestas del Plan. Entre estos se encuentra contar con las habilidades requeridas para la planificación e implementación del cambio (análisis cuantitativo; planificación y monitoreo) así como disponer de información diversa para la gestión (progreso de la implementación de la estrategia; referida a costos reales de las actividades, entre otros). Para la etapa de documentación, plantean la necesidad de contar con una declaración operacional, la cual incluye el programa de actividades o proyectos a realizar, los responsables, los hitos que son importantes de identificar y las metas propuestas (finales y de medio término). Asimismo, sugieren contar con un plan financiero y de recursos, que se vincule de manera explícita con el plan de operaciones. Finalmente, para la etapa de monitoreo, plantean

11 Evaluación y seguimiento del plan. 
que junto con disponer de información oportuna y de calidad para su adecuada ejecución, debe supervisar tanto el plan estratégico como los otros planes subsidiarios derivados de éste, abordando para ello distintos ámbitos temáticos, tales como: avance e impacto del proyecto general y de los proyectos específicos; seguimiento del financiamiento; cumplimiento del presupuesto; gestión de recursos; y calidad académica.

En consecuencia, los criterios propuestos para evaluar las capacidades de seguimiento de un plan consideran que:

a. La unidad cuente o pretenda desarrollar (en forma subsidiaria o propia) una capacidad de gestión de información y análisis que le permita evaluar el impacto, realizar el seguimiento de las actividades propuestas y monitorear la calidad de las principales actividades que desempeña.

b. Considere explícitamente el seguimiento y evaluación de su impacto en forma periódica, dando cuenta de ello a la comunidad.

c. Proponga un conjunto de mecanismos de aseguramiento de la calidad que permitan su monitoreo en el tiempo, promoviendo su mejora y evitando su detrimento.

\subsubsection{Fundamentos de la estrategia y ventajas sostenibles}

Dos publicaciones hacen mención a las posibles fuentes del valor de la institución, es decir, cuáles son los fundamentos sobre los cuales su estrategia se soporta. Con esto no se quiere negar la gravitación que el contenido de un plan y sus mecanismos de seguimiento tienen en el desempeño futuro de la institución, sino que se quiere destacar el hecho de que contar con un fundamento coherente de la estrategia podría significar un estándar de calidad superior del proyecto en cuestión.

Lynch \& Baines (2004) proponen la aplicación del sistema RBV (resourced-based view) para IES. Este concepto busca identificar conjuntos de recursos especiales de la organización que le permiten lograr un desempeño superior y establecer una ventaja competitiva sustentable, es decir, ella se hace difícil de ser imitada o sustituida y no es negociable por otra institución. 
Los argumentos utilizados por los autores para proponer la aplicación del RBV en IES, es que los recursos propios y únicos de la institución, más que su posición en el mercado, es lo que le permite contar con una ventaja competitiva sustentable (Dierickx \& Cool, 1989; Prahalad \& Bettis, 1986; Wernerfelt, 1984; Barney, 1986; Amit \& Schoemaker, 1993; Rumelt, 1991; Peteraf, 1993).

Para estos efectos, Lynch \& Baines (2004) deben demostrar la existencia de tres supuestos en las IES que permiten la aplicación del RBV:

- Suposición de mercado competitivo: las IES compiten por recursos de investigación, docencia y de cuerpos externos ${ }^{12}$.

- Reemplazo del supuesto de maximización de utilidades: si bien las IES no son instituciones con fines de lucro, los márgenes de utilidades permiten financiar importantes programas de inversión en las universidades y, por tanto, potenciar su desarrollo futuro.

- Significado de paquetes de ventajas competitivas en el ambiente de las IES

A continuación se analizan los supuestos necesarios para la aplicación del RBV para el caso chileno:

- Mercado competitivo: en Chile, casi la totalidad de los recursos de investigación son distribuidos mediante mecanismos concursables o provenientes de instituciones privadas que resguardan con celo su valor patrimonial. Hasta el momento, existe una alta competencia por atraer los buenos alumnos con incentivos como el Aporte Fiscal Indirecto, AFI, y cada vez más se flexibilizan los mecanismos de financiamiento estudiantil, lo cual hace más competitivo el sistema por la oferta de programas académicos; los empleadores son sólo leales a rentabilizar lo más posible su gasto y existen sistemas de head hunters y colocaciones bastante extendido.

- La suma de los recursos entregados por el Estado y los percibidos por las universidades a partir de sus matrículas y otras fuentes son bajos, por lo cual la necesidad de realizar una gestión eficiente es fundamental para sostener los programas de inversiones y

$12 \mathrm{Al}$ menos en las IES del Reino Unido. 
desarrollo que estas instituciones requieren. Como ejemplo, se puede mencionar que Chile, en términos de porcentaje del PIB, invierte menos de la mitad en las IES de lo que lo hacen los países desarrollados (OCDE, 2009).

- En el actual sistema universitario chileno es clara la relevancia de ciertas ventajas competitivas como lo es la planta de profesores, su perfeccionamiento, los núcleos de investigación, la calidad de los alumnos que ingresan y la experiencia acumulada.

Posteriormente, Lynch \& Baines (2004), basados en publicaciones anteriores (Kay, 1993; Prahalad \& Hamel, 1990), hacen una propuesta para la identificación de recursos competitivos sustentables:

- Reputación: la organización es capaz de comunicar información favorable sobre ella a sus stakeholders.

- Arquitectura: la red de relaciones contratos y alianzas.

- Capacidad de innovación: la habilidad de emprender nuevas iniciativas que van más allá de la actual estrategia.

- Competencias centrales: grupo de habilidades y tecnologías que permiten a la institución entregar un particular beneficio a sus clientes.

- Ventajas basadas en el conocimiento: propiedad tácita y explícita que la institución tiene sobre el conocimiento

Como se puede observar, las personas resultan ser un recurso fundamental, en tanto depositario y gestor de los grupos de ventajas competitivas presentadas.

Por su lado, Conway, Mackay, \& Yorke (1994) destacan la importancia de identificar los clientes y los caminos para satisfacerlos adecuadamente. Ya lo proponía Pearce en el año 1982, que la misma Misión debiera reconocer la demanda legítima de los stakeholders (Pearce, 1982).

Conway, Mackay, \& Yorke (1994) hacen el contrapunto entre el marketing con orientación al producto y aquél con orientación al servicio, exigiéndole a este último un involucramiento con los clientes y sus expectativas. En especial cuando la compra se realiza una vez en 
la vida, el "producto" es un intangible y tiene varios costos adicionales al dinero pagado. El paper declara la importancia de los stakeholders en el proceso de planificación estratégica, al punto de depender de ello la sobrevivencia de la IES (Thomas, 1980). En el caso de los alumnos, cobra más importancia la atención en ellos, toda vez que se identifican tanto como clientes así como parte de la oferta que las IES hacen a los empleadores y la sociedad en general (Lovelock \& Rothschild, 1980).

Por otra parte, Conway, Mackay \& Yorke (1994), dada la exposición a un ambiente cada vez más competitivo, plantean la necesidad de que la estrategia esté fundamentada en un conjunto de propuestas de valor relevantes para los stakeholders. Toda vez que en el ámbito de la educación superior una oferta valiosa para ellos permite generar círculos virtuosos y sinérgicos, el mantener y acrecentar esta relación será considerada como fuente de sustentabilidad de ventajas competitivas.

De esta forma, la propuesta de elementos y criterios de evaluación, en relación con las ventajas competitivas que fundamentan y sustenta la estrategia, formula la necesidad que el respectivo plan: a. Proponga el desarrollo de los recursos que son considerados estratégicos para la unidad, es decir, que son difíciles de imitar o replicar.

b. Establezca una relación permanente y de valor con sus stakeholders, y que, en general, las acciones y estrategias sean coherentes con este anhelo.

c. Plantee el desarrollo de relaciones interdisciplinarias con otras unidades académicas de la universidad y el establecimiento de convenios o acuerdos con instituciones nacionales o extranjeras, ya sea para el intercambio de alumnos, la obtención de becas, la formación de núcleos de investigación, entre otros.

d. Capitalice el conocimiento desarrollado tanto en la gestión de la docencia, como en la investigación y la oferta de servicios. Desarrolle sus capacidades de innovación en la docencia, generar publicaciones y patentes a partir de lo que investiga, aprovechar su relación con instituciones externas para el apoyo de la enseñanza, la venta de servicios y el desarrollo de investigación aplicada. 
e. Advierta propuestas innovadoras y una capacidad permanente de innovación más allá de las concretas.

\section{Comentarios finales y conclusiones}

La bibliografía muestra, en general, un consenso sobre los aspectos y ámbitos de un plan estratégico. Sin embargo, son pocas las referencias que destacan con claridad aquellos que son realmente importantes.

Una de las excepciones son Boyne \& Gould-Williams (2003), quienes en su investigación han definido la importancia de la capacidad predictiva de los elementos y contenidos de un plan estratégico para lograr un buen desempeño. Es así como demuestran que la actitud de los miembros hacia el Plan es el factor más relevante. Sin embargo, es necesario reflexionar que esa actitud y entusiasmo deben ser encauzados y sistematizados para obtener los objetivos que se proponen. Es decir, esta demostración de correlación (o no correlación) con el nivel de éxito futuro del proyecto no necesariamente invalida la necesidad de contar con elementos prácticos que permiten implementarlo y organizar los esfuerzos de quienes integran la institución.

Esta propuesta muestra un conjunto razonable de criterios de calidad, no obstante, no debemos olvidar que este trabajo nace de una necesidad real y práctica, por lo que varios elementos que podrían haber sido desechados en el estudio de la bibliografía son importantes para la forma de gestión y gobierno de una universidad compleja.

Estos criterios sólo lograrán ser evaluados en el contexto de su uso y, como producto de la experiencia, tenderán a su mejoramiento continuo. Incluso, a partir de dicha información, podrían iniciarse estudios orientados a eliminar variables que pudieran ser redundantes, así como proponer nuevas que actualmente se encuentren excluidas. 


\section{Referencias bibliográficas}

Amit, R., \& Schoemaker, P. (1993) Strategic assets and organizational rent. Strategic Management Journal, vol. 14, pp. 33-46.

Andersen, T. J. (2000) Strategic Planning, Autonomous Actions and Corporate Performance. Long Range Planning, 33, pp. 184-200.

Armstrong, J. (1982) The Value of Formal Strategic Planning for Strategic Decisions: Review of Empirical Research. Strategic Management Journal, vol. 3 (n. ${ }^{\circ}$ ), pp. 197-211.

Barney, J. B. (1986) Organizational culture: Can it be a source of sustained competitive advantage? Academy of Management Review, vol. 11, pp. 656-665.

Bower, J. (1970/1982) Managing the Resource Allocation Process. Boston, MA: Harvard Business School Press.

Boyd, B. K., \& Reuning-Elliott, E. (1998) A Measurement Model of Strategic. Strategic Management Journal, vol. 19 (n. ${ }^{\circ} 2$ ), pp. 181-192.

Boyne, G., \& Gould-Williams, J. S. (2003) Planning and Performance in Public Organizations. Public Management Review, vol. 5 (n. ${ }^{\circ}$ 1), pp. 115-132.

Burgelman, R. (1983) A model of the interaction of strategic behavior, corporate context, and the concept of strategy. Academy of Management Review, vol. 8, pp. 61-70.

Burgelman, R. (1988) Strategy making as a social learning process: the case of internal corporate venturing. Interfaces, vol. 18, pp. 74-85.

Camillus, J. (1975) Evaluating the benefits of Formal Planning Systems. Long Range Planning, vol. 8 (n. ${ }^{\circ}$ ), pp. 33-40.

Capon, N., Farley, J., \& Hubert, J. (1987) Corporate Strategic Planning. Columbia University Press.

Chandler, A. (1962) Strategy and Structure. MIT Press.

Conway, T., Mackay, S., \& Yorke, D. (1994) Strategic Planning in Higher Education: Who Are the Customers? International Journal of Educational Management, vol. 8 (n. ${ }^{\circ}$ ), pp. 29-36.

Dierickx, I., \& Cool, K. (1989) Asset stock accumulation and sustainability of competitive advantage. Management Science, vol. 35, pp. 1504-1511.

HEFCE Audit Service. (Jun de 2000) Strategic planning in higher education: a guide for heads of institutions, senior managers and members of governing bodies. HEFCE publications. HEFCE.

Kay, J. (1993) Foundations of Corporate Success. Oxford University Press. 
Kotler, P. \& Murphy, P. E. (1981) Strategic Planning for Higher Education. (O. S. Press, Ed.) The Journal of Higher Education, vol. 52, n. ${ }^{\circ}$, pp. 470-489.

Leach, S. (1982) In Defence of the Rational Model. (S. L. Stewart, Ed.) Approaches in Public.

Lovelock, C. \& Rothschild, M. (1980) Uses, Abuses and Misuses of Marketing in Higher Education (T. C. Board, Ed.) Marketing in College Admissions: A Broadening of Perspectives.

Lynch, R. \& Baines, P. (2004) Strategy Development in UK Higher Education: Towards Resource-based Competitive Advantages. Journal of Higher Education Policy and Management, vol. 6 (n. ${ }^{\circ}$ 2), pp. 171-187.

Mintzberg, H. (1994) The Fall and Rise of Strategic Planning. Harvard Business Review, vol. 72, pp. 107-114.

Noda, T. \& Bower, J. (1996) Strategy making as iterated processes of resource allocation. Strategic Management Journal, vol. 17, pp. 159-192.

Normann, R. \& Pennings, J. (1985) Organizational Strategy and Change: New Views on Formulating and Implementing Strategic Decisions. San Francisco, CA: Jossey-Bass.

OCDE (2009) La Educación Superior en Chile, Revisión de Politicas Nacionales de Educación.

Pearce, J. (1982) The Company Mission as a Strategic Tool. Sloan Management Review (Spring 1982), pp. 15-24.

Peteraf, M. A. (1993) The Cornerstones of Competitive Advantage: A Resourcebased View. Strategic Management Journal, vol. 14, pp. 179-191.

Prahalad, C. K. \& Bettis, R. A. (1986) The Dominant logic: A New Linkage Between Diversity and Performance. Strategic Management Journal, vol. 7, pp. 485-501.

Prahalad, C. K. \& Hamel, G. (1990) The Core Competence of the Corporation. Harvard Business Review, May/June, pp. 79-91.

Rumelt, R. P. (1991) How Much Does Industry Matter? Strategic Management Journal, vol. 12, pp. 64-75.

Self, P. (1974) Is Comprehensive Planning Possible and Rational? Policy and Politics, vol. 2 (n. ${ }^{\circ}$ ), pp. 193-203.

Shirley, R. (1983) Identifying the Levels of Strategy for a College or University. Long Range Planning, vol. 16 (n. ${ }^{\circ}$ 3), pp. 92-98. 
Streib, G. \& Poister, T. (1990). Strategic Planning in US Cities: Patterns of Use, Perceptions of. American Review of Public Administration, vol. 20 (n. $\left.{ }^{\circ} 1\right)$, pp. $29-44$.

Thomas, R. (1980) Corporate Strategic Planning in a University. Long Range Planning, vol. 13, pp. 70-78.

Wernerfelt, B. (1984) A Resource-based View of the Firm. Strategic Management Journal, vol. 5, pp. 171-180.

Wildavsky, A. (1973) If Planning Is Everything, Maybe It's Nothing. Policy Sciences, vol. 4, pp. 127-153.

Recibido: 17/08/2009

Aceptado: 26/04/2010 